\title{
Insights into Carbon Nanotube-Metal Oxide Composite: Embedding in Membranes
}

\author{
Tawfik A Saleh* \\ Chemistry Department, King Fahd University of Petroleum \& Minerals, Dhahran, Saudi Arabia
}

Received: July 21, 2017; Accepted: September 28, 2017 ; Published: November 27, 2017

*Corresponding author: Tawfik A Saleh, Chemistry Department, King Fahd University of Petroleum \& Minerals, Dhahran, Saudi Arabia, Tel: +966 13 860 1734; E-mail: tawfik@kfupm.edu.sa

\begin{abstract}
The fields of the nanocomposites and membranes have received a lot of attention and close examination of scientists and engineers in recent years. This makes it possible to design and create new materials with unprecedented flexibility. The driving force behind the fabrication of novel nanocomposites is to achieve high functional properties for high-end applications. This review provides an overview and insights into carbon nanotube-metal oxide composites and the same embedded in membranes. The current state of research has indicated the potential of these nanocomposites due to their improving properties. Fundamental work in processing, characterization, and analysis is important before the structural properties of this class of materials are also of interest.
\end{abstract}

Keywords: Nanocomposites; Carbon Nanotube; Functional; Membrane;

\section{Introduction}

Since their discovery [1], carbon Nanotubes (CNTs) has been intensively investigated due to their unique mechanical, chemical and physical properties. There are two main types of carbon nanotubes; Single-Walled Nanotubes(SWNTs) consist of single graphite sheets wrapped into a cylindrical tube, and Multiwalled Nanotubes(MWNTs) comprise an array of such nanotubes concentrically nested like rings of a tree trunk [2]. Many potential applications have been proposed, including sensors, probes, and energy and hydrogen storage device. Also, CNT and its matrix have been investigated for water treatment in sorption, fixed bed mode, and membrane.

The major drawback in the extensive use of membranes includes membrane fouling, which results in flux decline during operation [4]. Several types of fouling could occur in the membrane system, e.g. crystalline fouling, organic fouling, particulate and colloidal fouling, and microbial fouling, etc. [4]. Many approaches to control membrane fouling have been performed, which generally involve pre-treatment of the feed solution, surface modification of the membrane (like hydrophobic or hydrophilic and electronegative or electropositive modification), optimization of module arrangement and process conditions, and periodic cleaning [5]. Even after the development for decades, particulate and colloidal fouling still remains the main reason for flux decline in the process of industrial wastewater treatment.

\section{Carbon nanotube modified membrane}

The synthesis of CNT/polymer membrane structures has attracted growing interest and a number of methods to produce such membranes have emerged in recent years. The synthesis of such materials is achieved by chemical modification of the graphitic sidewalls and tips to enhance their chemical affinity to polymer matrices [6]. Various chemical strategies for grafting polymers onto carbon nanotubes and the manufacturing of carbon nanotube/polymer nanocomposites have been reported with enhanced mechanical and chemical properties of the membranes. Because of the excellent mechanical properties of CNTs, incorporation of CNTs into a polymer matrix leads to structural materials with significantly high modulus and strength. Significant advancement has been made in improving the mechanical properties of polymer matrix by CNTs with a polymer to form membranes.

An example is the fabrication of CNT/ polymer composite membranes with good interface adhesion and good nanotube dispersion as shown in Figure 1. Also, CNT can be used to modify poly (vinyl alcohol) (PVA) membranes by one-step route for the incorporation of CNT into PVA. The success of the method can be approved by several characterization techniques such as molecular-level insights of morphological changes of polymer

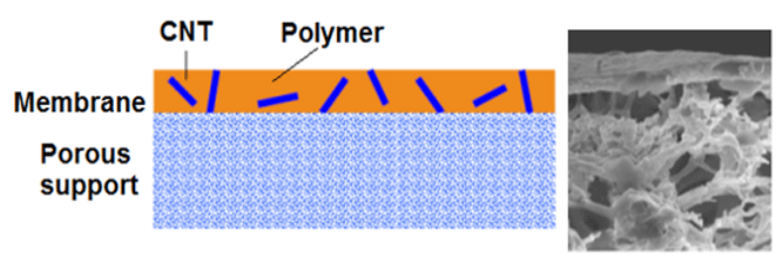

Figure 1: CNT/ polymer composite membranes

chains due to nanotube incorporation, as shown in Figure 2. Other instruments can be used including transmission Electron Microscope (TEM), Field Emission Scanning Electron Microscope (FESEM), Energy Dispersive X-ray (EDX), Raman spectroscopy, 
Thermogravimeter (TGA), and Fourier Transformed-Infrared Spectroscopy (FT-IR), X-ray Photoelectron Spectroscopy (XPS), and nuclear magnetic resonance spectroscopy, most commonly known as NMR spectroscopy or Magnetic Resonance Spectroscopy (MRS).

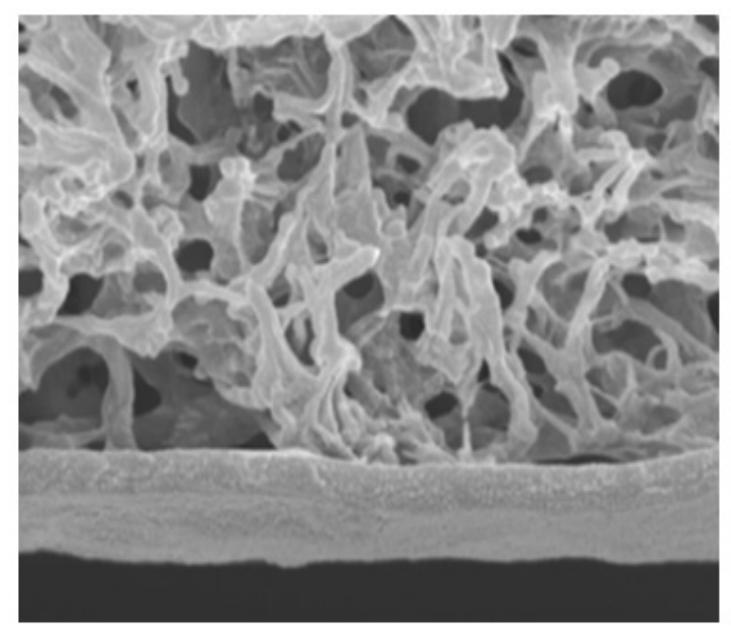

Figure 2: SEM image of CNT modified poly(vinyl alcohol) (PVA) membrane

\section{Nanoparticles modified membrane}

Preparation of organic-inorganic composite membranes is a promising approach to reduce void formation and improve the mechanical strength of polymer membranes. Metal oxides Nanoparticles (NP), such as titania and alumina loaded on carbons, were considered for preparing membranes for water applications. The effects of nanoparticle loading, size and morphology, and methods of nanoparticle incorporation on the functional properties of the resulting nanocomposite membranes have been studied. There are several methods of preparing NP/ membranes, including phase inversion, coagulation, precipitation and interfacial polymerization, in addition to a combination of solvent casting and melt mixing method.

Titanium $\left(\mathrm{TiO}_{2}\right.$ ) has been the focus of numerous investigations in recent years, because of its high hydrophilicity, stable chemical property, and low cost, etc. Most of researchers carried out in this field have focused on the use of $\mathrm{TiO}_{2}$ powder suspended in the water as a catalyst. The method of $\mathrm{TiO}_{2}$ self-assembly on the surface with the terminal functional groups (for example, single crystal silicon, quartz, and glass substrates) has been used to fabricate multilayer ultrathin films. However, the cost of NPs and their availability remain the significant obstacles to the application of NPs for water treatment. A significant reduction in flux loss was observed upon compaction for porous, phase inversion, $\mathrm{TiO}_{2}$ nanoparticle filled membranes.

Increasing loadings of alumina $\left(\mathrm{Al}_{2} \mathrm{O}_{3}\right)$ nanoparticles in phase inversion PVDF membranes were correlated with an increase in hydrophilicity of the modified membranes, while incorporation of $\mathrm{TiO}_{2}$ nanoparticles to phase inversion membranes ( $\mathrm{Li}$, et al, 2006) showed hydrophilicity enhancement $[7,8]$.

\section{Carbon Nanotube Loaded With Nanoparticles}

CNTs decorated with Metal Oxide (MO) Nanoparticles (NP) form a new class of hybrid nanomaterials that could potentially display not only the unique properties of NPs and nanotubes but also additional novel physical and chemical properties due to the interaction between CNTs and attached NPs [13-24].

Many studies have been devoted to the fabrication of metal nanoparticles-decorated CNTs for unique chemical, physical and optical properties. Various composites of MWCNT with metal oxides such as alumina, tungsten oxide, and titanium dioxide were reported [9-12]. Also, we have reported the successful encapsulation of iron oxide in carbon nanotubes, yielding a new type of hybrid nanostructure that combines all the key magnetic properties of the guest molecules with the functional properties of the host nanotube. The fabricated nanocomposites have been tested for different applications related to water treatment; sorption and photodegradation. MWCNT/ $\mathrm{WO}_{3}$ showed enhanced photocatalytic activity toward dyes degradation under solar irradiation comparing with $\mathrm{WO}_{3}$ nanoparticles. The enhancement in photocatalytic performance has been explained based on adsorption ability and electron transportation as a result of a strong interaction between $\mathrm{WO}_{3}$ and MWCNTs. Other example used as photocatalyst for phenol degradation under irradiation of visible light, is $\mathrm{MWCNT}_{\mathrm{TiO}}$, Figure 3, which showed higher efficiency compared to pure $\mathrm{TiO}_{2}$. Also, $\mathrm{CNT} / \mathrm{TiO}_{2}$ showed high activity for the decomposition of dyes and alcohol in water under UV irradiation. However, the large-scale practical application of CNT/MO nanocomposites will be difficult to realize if such materials have been used in suspension systems or in fixed bed column mode for water treatment.

Based on the literature review as well as our experience, we share with the researchers and scientists the idea of encapsulation CNT/MO nanocomposites aiming to address the one major challenge of membrane used in water treatment units, namely efficient membrane, which tolerates harsh conditions such as high pressure and temperature. This is due to the fact that CNT and MO have high mechanical and thermal properties as well as chemical and physical stability. The objective is to produce a CNT/MO-polymer nanocomposite membrane with enhanced properties. The membrane is expected to have the properties of each component; CNT, NP and polymer, and additional novel properties due to the interaction between them. For the implementation of the idea, CNT/MO composites first to be synthesized. Then, this composite can be embedded in a membrane. Chemical interaction between the composite and the polymer is of importance. In addition, fundamental understanding of the binding mechanism between them will facilitate the rational design and use of these membranes.

This is with taken into consideration that membrane fouling is still a critical problem for efficient commercialization of filtration plants, which results in flux decline with operating time. Therefore, application of the resulted membrane for water treatment is expected to reduce fouling. This is because, CNTs can enhance the mechanical stability which is of major importance 

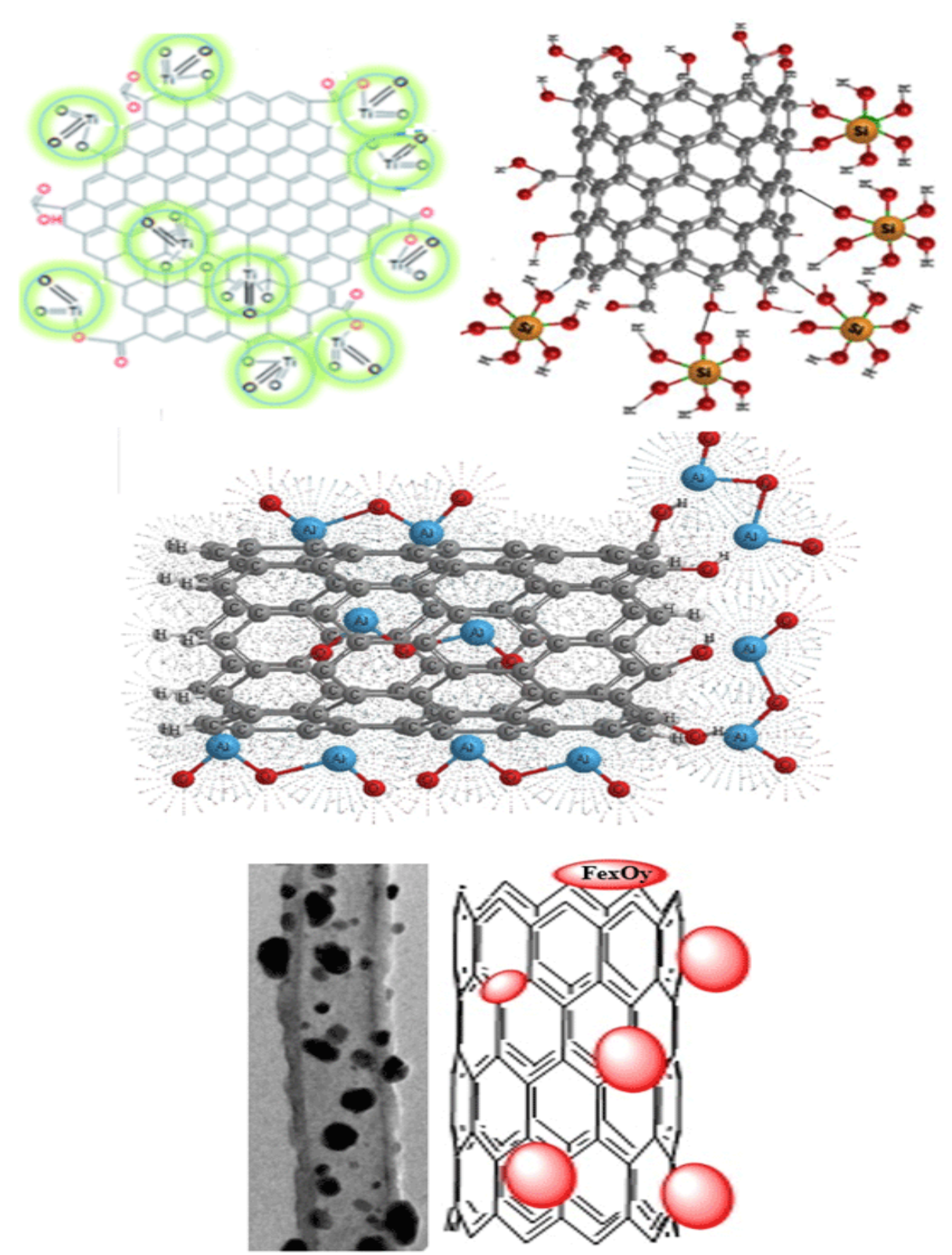

Figure 3: Examples of metal oxides loaded on carbon structures; titania, alumina, silica and iron oxides loaded on carbons

for membranes, as it is required to prevent structural breakage at high pressures and thus ensure the membrane's operational viability, thus, improvement of the mechanical properties of polymeric membranes via nanocomposites incorporation into polymer [13].

Titania of photocatalytic destructive capability on microorganisms is considered to be a novel mean to reduce membrane biofouling, which is considered the main reason for flux decline. Therefore, embedding CNT/TiO2 nanocomposite in a membrane is enhancing the permeability over a prolonged period of reverse osmosis operation. Silver nanoparticles are considered to be effective in deactivating the microorganism.
Thus, embedding CNT/Ag composite in a membrane is expected to increase anti-biofouling function in order to reduce the contaminants decreasing the efficiency of the membrane. The presence of NPs is also expected to improve the compaction resistance of the membranes. The hydrophilicity can be highly controlled by functionalizing CNT with either hydrophilic or hydrophobic group. Thermal stability and flammability can be enhanced by embedding CNT/silica oxide nanocomposites. The nanocomposites embedded into membrane can be used in water treatment, purification. Such membranes can also be used in different applications. The idea allows for the more robust design of nanocomposite membrane properties as well. 


\section{Conclusion}

Incorporation of nanoparticles into a forming membrane matrix appears to be an attractive approach to the design of nanocomposite structures with improved mechanical and separation properties and with embedded nanoparticle-based functionalities. It is important to note that work reported to date has utilized multiwalled nanotubes CNT with a variety of different structures, with the choice of CNT dictated by availability or processing considerations. Properties that do not rely on high synergy between the nanoparticle and CNTs, such as electrical conductivity, have already shown notable enhancements. However, the chemical interaction between nanoparticles and CNT is not yet clear since CNT has many functional groups. In addition, the embedding of CNT or nanoparticles-modified CNT within membranes is an interesting area of research that requires further investigation.

\section{Acknowledgment}

The authors would like to acknowledge the King Fahd University of Petroleum \& Minerals (KFUPM) for the encouragement.

\section{References}

1. Iijima S. Helical microtubules of graphitic carbon. Nature. 1991;354:5658. doi:10.1038/354056a0

2. RH Baughman, AA Zakhidov, WA de Heer. Carbon nanotubes--the route toward applications. Science. 2002;297(5582):787-792. DOI: 10.1126/science.1060928

3. Y Wang, JH Kim, KH Choo, YS Lee, CH Lee, Hydrophilic modification of polypropylene microfiltration membranes by ozone-induced graft polymerization. J Membr Sci. 2000;169(2): 269-276. doi.org/10.1016/ S0376-7388(99)00345-2

4. HC Flemming. Reverse osmosis membrane biofouling. Exp Therm Fluid Sci. 1997;14(4):382-391. doi.org/10.1016/S0894-1777(96)00140-9

5. Tawfik A. Saleh. Nanocomposite of carbon nanotubes/silica nanoparticles and their use for adsorption of Pb (II): from surface properties to sorption mechanism.Desalination and Water Treatment. 2015;57:10730-10744. doi.org/10.1080/19443994.2015.1036784

6. Spitalsky Z, Tasis D, Papagelis K, Galiotis C. Carbon Nanotube-Polymer Composites: Chemistry, Processing, Mechanical and Electrical Properties.Progress in Polymer Science. 2010;35:357-401. doi. org/10.1016/j.progpolymsci.2009.09.003

7. LYan, YS Li, CB Xiang, S Xianda. Effect of nano-sized Al203-particle addition on PVDF ultrafiltration membrane performance. J Membr Sci. 2006;276:162-167. doi.org/10.1016/j.memsci.2005.09.044

8. JB Li, JW Zhu, MS Zheng. Morphologies and properties of poly(phthalazinone ether sulfone ketone) matrix ultrafiltration membranes with entrapped TiO2 nanoparticles. J Appl Polym. Sci.2006;103(6):3623-3629.DOI: 10.1002/app.25428
9. TA Saleh, VK Gupta. Functionalization of tungsten oxide into MWCNT and its application for sunlight-induced degradation of rhodamine B. J Colloid Interface Sci. 2011;362(2):337-344. doi.org/10.1016/j. jcis.2011.06.081

10. TA Saleh, S Agarwal, VK Gupta. Synthesis of MWCNT/MnO 2 and their application for simultaneous oxidation of arsenite and sorption of arsenate. Applied Catalysis B:Environmental. 2011;106(1-2):46-53. doi.org/10.1016/j.apcatb.2011.05.003

11. Tawfik A. Saleh. Nanotechnology in Oil and Gas Industries: Principles and Applications. 2017.

12. V. K. Gupta, S. Agarwal, T. A. Saleh.Synthesis and characterization of alumina-coated carbon nanotubes and their application for lead removal. J Hazard Mater. 2011;185(1):17-23. doi: 10.1016/j. jhazmat.2010.08.053

13. R Van Noorden. The trials of new carbon. Nature. 2011;469:14-16. DOI: $10.1038 / 469014 a$

14. Tawfik A. Saleh. Mercury sorption by silica/carbon nanotubes and silica/activated carbon: a comparison study. Journal of Water Supply: Research and Technology-Aqua. 2015;64(8):892-903. DOI: 10.2166/ aqua.2015.050

15. VK Gupta, Tawfik A. Saleh. Syntheses of Carbon Nanotube-Metal Oxides Composites; Adsorption and Photo-degradation. DOI: 10.5772/18009

16. Tawfik A Saleh, VK Gupta. Nanomaterial and Polymer Membranes: Synthesis, Characterization, and Applications. 2016.

17. Tawfik A Saleh. Applying Nanotechnology to the Desulfurization Process in Petroleum Engineering. 2016. DOI: 10.4018/978-1-46669545-0

18. Tawfik A Saleh. Advanced Nanomaterials for Water Engineering, Treatment, and Hydraulics. 2017. DOI: 10.4018/978-1-5225-2136-5

19. VK Gupta, S Agarwal, T A Saleh. Chromium removal by combining the magnetic properties of iron oxide with adsorption properties of carbon nanotubes. Water Research. 2011;45(6):2207-2212. doi. org/10.1016/j.watres.2011.01.012

20. Tawfik A Saleh. The influence of treatment temperature on the acidity of MWCNT oxidized by HNO3 or a mixture of HNO3/H2SO4, Applied surface science. 2017;257(17):7746-7751.

21. Tawfik A Saleh. Isotherm, kinetic, and thermodynamic studies on Hg (II) adsorption from aqueous solution by silica-multiwall carbon nanotubes.Environmental Science and Pollution Research. 2015;22(21):16721-16731. DOI:10.1007/s11356-015-4866-z

22. Tawfik A Saleh. The role of carbon nanotubes in enhancement of photocatalysis. 2013. DOI: $10.5772 / 51050$

23. I Ali, MR Akl, GA Meligi, Tawfik A Saleh. Silver nanoparticles embedded in polystyrene-polyvinyl pyrrolidone nanocomposites using $\gamma$-ray irradiation: Physico-chemical properties. Results in Physics. 2017;7:1319-1328. doi.org/10.1016/j.rinp.2017.03.028

24. Tawfik A Saleh. Activated carbon/aluminum oxide/polyethylenimine composites and methods thereof. 2017. 